\title{
EDITORIAL
}

\section{The COPD CO-factor}

\author{
G.P. Anderson
}

I $\mathrm{n}$ the current issue of the European Respiratory Journal, BATHOORN et al. [1] present findings that may open up new avenues of treatment for chronic obstructive pulmonary disease (COPD) and provide fundamental insights into the pathogenesis of the condition. BATHOORN et al. [1] present a pilot study on the effect of inhaled carbon monoxide (CO) on inflammation and methacholine responsiveness in COPD.

In their study, 22 ex-smokers with stable COPD (forced expiratory volume in one second (FEV1) $>1.2 \mathrm{~L}, \mathrm{FEV} 1 /$ forced vital capacity $<70 \%$ predicted) were administered 100 125 ppm CO, a low concentration, for $2 \mathrm{~h}$ a day over 4 days in a randomised, cross-over protocol [1]. Maximal blood carboxyhaemoglobin peaked at $4.5 \%$, well within safe levels. Eosinophil counts, but not other cells, followed a downward trend in induced sputum, while methacholine responsiveness was slightly and significantly improved. The study was designed and performed with careful attention to ethics and patient safety. However, it is noteworthy that two of the patients experienced exacerbations of COPD during the $\mathrm{CO}$ treatment arm.

The effects of $\mathrm{CO}$ were weak, but tangible, and not uniformly statistically significant. Yet, in several ways this pilot study is intriguing. Why? Because the paper points to the potential therapeutic utility of inhaled $\mathrm{CO}$, a known respiratory system poison that is abundant in tobacco smoke, the cause of most COPD. In addition to this, endogenously generated $\mathrm{CO}$ has been discovered by other authors in exhaled breath and blood in COPD [2, 3], and recent fundamental basic research has revealed that endogenous $\mathrm{CO}$ is an important physiological regulatory factor in systems as diverse as smooth muscle and the immune system. Like nitric oxide (NO), $\mathrm{CO}$ is emerging as a major, and increasingly complex, modulator with potentially beneficial and detrimental biological effects.

The study by BATHOORN et al. [1] already seems to hint at this diversity. One way to understand the cryptic meanings in the study is to consider that delivering exogenous CO may simply intensify the effects of endogenous $\mathrm{CO}$ already being made at increased concentrations in the COPD lung. Endogenous CO is formed during the catabolism of haem, by haemoxygenase (HMOX, but more commonly abbreviated as HO) enzymes

Lung Disease Research Group, Depts of Medicine and Pharmacology, University of Melbourne, Victoria, Australia

STATEMENT OF INTEREST: None declared.

CORRESPONDENCE: G.P. Anderson, Lung Disease Research Group, Depts of Medicine and Pharmacology, University of Melbourne, Victoria, Australia. Fax: 61 383440241. E-mail: gpa@unimelb.edu.au producing bilirubin and other derivatives as co-products. HMOX1 (HO-1) is inducible, whereas HMOX2 (HO-2) is constitutively expressed. Good evidence links the HMOX system to COPD. In experimental animal models of COPD, and in smokers, oxidants in cigarette smoke strongly induce HO-1 where it is likely to play a protective role. Transcriptional efficiency of HMOX is regulated, in part, by the (GT)n dinucleotide repeat in the 5'-flanking region of the gene; the greater the number of repeats the weaker the gene induction. A long dinucleotide repeat sequence has been linked to emphysema susceptibility in smokers [4], lung adenocarcinoma (a common cancer in COPD patients) and rate of decline of lung function $[5,6]$. Cell culture studies have demonstrated that HMOX protects cells from oxidative stress. EGR-1, the early growth response- 1 transcription factor, found to differentiate Global Initiative for Chronic Obstructive Lung Disease (GOLD) II and GOLD 0/at-risk smoker gene profiles, controls expression of HMOX1 amongst other genes [7]. In early disease, elevated $\mathrm{CO}$ might be protective by reducing net oxidant load.

The situation in established COPD is less certain. A critical step in COPD pathogenesis seems to be the point at which antioxidant defences become uncoupled from, or overwhelmed by, oxidant load. This may be why in established COPD, CO levels rise in parallel with disease severity and biochemical and biomarkers of oxidative stress, and are associated with elevated acute phase protein in the blood [3].

Co-regulation of oxidative stress is, however, unlikely to be the only role of $\mathrm{CO}$. $\mathrm{CO}$ has been demonstrated to protect cells from apoptosis and to exert anti-inflammatory and antiproliferative effects in a range of in vivo and in vitro cells models $[8,9] \mathrm{CO}$ also protects against reperfusion injury and acute lung injury, an effect linked to reduced activation of extracellular signal regulated kinase (ERK) which, since ERKs help activate transcription involving activator protein-1, would explain reduced inflammatory gene expression [8].

In the nervous system, $\mathrm{CO}$ is almost certainly a physiological neurotransmitter. $\mathrm{CO}$ mediates a large part of neuronal nonadrenergic/noncholinergic (NANC) airway smooth muscle relaxation in guinea pigs [10]. NANC relaxation is weak or absent in humans, but CO is an effective bronchodilator [11] and is much more effective than NO donors (e.g. nitrate vasodilators) even though both molecules activate the soluble guanylate cyclase/guanosine monophosphate transduction pathway. Understanding this difference alone could lead to new classes of bronchodilator. One clue comes from the recently developed drugs called CO-releasing molecules (CORMs). In other smooth muscles, CORMs induce CO 
formation and contribute to relaxation via ion channel opening. Thus, CO seems to belong to a growing family of endogenous negative or "bronchoprotective" regulators of inflammation and airflow limitation, that have been identified in the lung. It also now seems obvious that endogenous $\mathrm{CO}$, and therapeutically administered $\mathrm{CO}$, must affect ventilation/perfusion mismatching, which is marked in COPD, by reducing both airway and vascular smooth muscle tone. In the study by BATHOORN et al. [1] only a slight reduction in methacholine sensitivity was observed.

While the study by BATHOORN et al. [1] was not powered to detect effects of $\mathrm{CO}$ on exacerbation rate or severity, it is of some concern that two exacerbations occurred in the $\mathrm{CO}$ treatment period. Exacerbations in COPD are frequently caused by viruses. Eosinophil number was reduced by $\mathrm{CO}$ but often increases during viral exacerbation in COPD in humans [12] and eosinophils may possibly exert an antiviral effect that could be blunted by CO. Similarly, bacteria are associated with at least one-third of COPD exacerbations. Tolllike receptors (TLRs) are needed for recognition and clearance of bacteria. In macrophages, $\mathrm{CO}$ has recently been shown to inhibit signalling by TLR2, 4, 5 and 9 (but not TLR3) [13]. Here the mechanism of $\mathrm{CO}$ is complex: it acts indirectly by suppressing the trafficking of TLRs into lipid-raft signalling complexes in the cell membrane. This is dependent, also indirectly, on reducing reactive oxygen species at the level of their generation by reduced nicotinamide adenine dinucleotide phosphate oxidase. There is now good evidence that $\mathrm{CO}$ suppresses allograft rejection [14] via immune suppression, anti-proliferative effects and protection against apoptosis. These effects are seen in model systems at higher concentrations, around $500 \mathrm{ppm}$, much higher than the conservative concentrations used in the study by BATHOORN et al. [1], and they need to be reconciled against the observation that $\mathrm{CO}$ concentrations rise spontaneously in acute exacerbations of COPD [2].

It will therefore be essential to determine whether the presumptive beneficial effects of $\mathrm{CO}$ observed in the study by BATHOORN et al. [1] (which might be better if the amount of $\mathrm{CO}$ were to be increased), can only be obtained at the cost of weakening defences against infective exacerbations. Many anti-inflammatory drugs being developed as potential treatment for COPD are marred by this risk. If endogenous $\mathrm{CO}$ is proven to suppress local immune defence, it may help to understand why COPD patients are susceptible to recurrent infectious exacerbations and highlight the need for $\mathrm{CO}$ suppressors, rather than inhaled $\mathrm{CO}$, as treatments.

At present, it seems reasonable to conclude that the HMOX-CO system may offer protection from COPD, at least early in the pathogenesis of the condition. It is much less clear whether elevated $\mathrm{CO}$ is of net benefit in established COPD. In particular, the ability of exogenous $\mathrm{CO}$ to suppress immune defences, such as the TLR systems in macrophages, suggests a possible risk that "therapeutically" administered CO might predispose to infection. Conversely, inhaled $\mathrm{CO}$, if it is proven to be safe and effective, already displays the highly desirable, and much sought after by the industry, profile of an antiinflammatory bronchodilator.
Given the clinical diversity of chronic obstructive pulmonary disease presentations, further work is needed before inhaled carbon monoxide can be thought of as a therapeutic agent. Perhaps carbon monoxide donors/inducers will find a role but suppressors may emerge as more important agents if exacerbation risk is worsened. In any case, the underlying research that will be needed to guide any clinical application of carbon monoxide will lead to major advances in understanding of the nature of chronic obstructive pulmonary disease and its pathogenesis. An exciting step forward has been taken by BATHOORN et al. [1], who have translated a large corpus of preclinical knowledge into the first human pilot study of carbon monoxide in chronic obstructive pulmonary disease.

\section{REFERENCES}

1 Bathoorn E, Slebos D-J, Postma DS, et al. Anti-inflammatory effects of inhaled carbon monoxide in patients with COPD: a pilot study. Eur Respir J 2007; 30: 1131-1137.

2 Yasuda H, Yamaya M, Nakayama K, et al. Increased arterial carboxyhemoglobin concentrations in chronic obstructive pulmonary disease. Am J Respir Crit Care Med 2005; 171: 1246-1251.

3 Montuschi P, Kharitonov SA, Ciabattoni G, et al. Exhaled 8isoprostane as a new non-invasive biomarker of oxidative stress in cystic fibrosis. Thorax 2000; 55: 205-209.

4 Yamada N, Yamaya M, Okinaga S, et al. Microsatellite polymorphism in the heme oxygenase-1 gene promoter is associated with susceptibility to emphysema. Am J Hum Genet 2000; 66: 187-195.

5 Nakayama K, Kikuchi A, Yasuda H, et al. Heme oxygenase-1 gene promoter polymorphism and decline in lung function in Japanese men. Thorax 2006; 61: 921.

6 Kikuchi A, Yamaya M, Suzuki S, et al. Association of susceptibility to the development of lung adenocarcinoma with the heme oxygenase-1 gene promoter polymorphism. Hum Genet 2005; 116: 354-360.

7 Ning W, Li C-J, Kaminski N, et al. Comprehensive gene expression profiles reveal pathways related to the pathogenesis of chronic obstructive pulmonary disease. PNAS 2004; 101: 14895-14900.

8 Brouard S, Otterbein LE, Anrather J, et al. Carbon monoxide generated by heme oxygenase 1 suppresses endothelial cell apoptosis. J Exp Med 2000; 192: 1015-1026.

9 Song R, Mahidhara RS, Liu F, Ning W, Otterbein LE, Choi AM. Carbon monoxide inhibits human airway smooth muscle cell proliferation via mitogen-activated protein kinase pathway. Am J Respir Cell Mol Biol 2002; 27: 603-610.

10 Dellabianca A, Sacchi M, Anselmi L, et al. Role of carbon monoxide in electrically induced non-adrenergic, noncholinergic relaxations in the guinea-pig isolated whole trachea. Br J Pharmacol 2007; 150: 220-226.

11 Cardell LO, Ueki IF, Stjarne P, et al. Bronchodilatation in vivo by carbon monoxide, a cyclic GMP related messenger. Br J Pharmacol 1998; 124: 1065-1068.

12 Papi A, Bellettato CM, Braccioni F, et al. Infections and airway inflammation in chronic obstructive pulmonary disease severe exacerbations. Am J Respir Crit Care Med 2006; 173: 1114-1121. 
13 Nakahira K, Kim HP, Geng XH, et al. Carbon monoxide differentially inhibits TLR signaling pathways by regulating ROS-induced trafficking of TLRs to lipid rafts. J Exp Med 2006; 203: 2377-2389.
14 Song R, Kubo M, Morse D, et al. Carbon monoxide induces cytoprotection in rat orthotopic lung transplantation via anti-inflammatory and anti-apoptotic effects. Am J Pathol 2003; 163: 231-242. 